Cipango Cahiers d'études japonaises

Hors-série | 2008

Autour du Genji monogatari

\title{
Un art de la citation
}

An Art of Quotation

\section{Sumie Terada}

\section{(2) OpenEdition}

Journals

Édition électronique

URL : https://journals.openedition.org/cipango/597

DOI : 10.4000/cipango.597

ISSN : 2260-7706

Éditeur

INALCO

Édition imprimée

Date de publication : 1 janvier 2008

Pagination : 133-155

ISBN : 978-2-85831-170-5

ISSN : 1164-5857

\section{Référence électronique}

Sumie Terada, « Un art de la citation », Cipango [En ligne], Hors-série | 2008, mis en ligne le 11 juin 2012, consulté le 30 juin 2021. URL : http://journals.openedition.org/cipango/597 ; DOl : https:// doi.org/10.4000/cipango.597

Ce document a été généré automatiquement le 30 juin 2021.

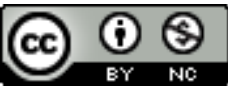

Cipango est mis à disposition selon les termes de la Licence Creative Commons Attribution - Pas d'Utilisation Commerciale 4.0 International. 


\title{
Un art de la citation
}

\author{
An Art of Quotation
}

Sumie Terada

\section{Le Roman du Genji au service du renga}

\section{Rayonnement des mots}

1 Vers la fin du XII siècle, soit deux siècles après la rédaction du Roman du Genji, Murasaki Shikibu intègre définitivement le panthéon de la poésie japonaise. Reconnu désormais comme référence incontestée, le Roman du Genji inspire non seulement d'autres écrits romanesques, mais à partir de cette période sert de modèle et de source d'inspiration pour la composition du waka'. L'intérêt que suscite ce roman s'accroît encore avec l'apparition du renga (vers en chaîne), forme poétique caractéristique de la période médiévale (XIII ${ }^{\mathrm{e}} \mathrm{XVI}{ }^{\mathrm{e}}$ siècles). En effet, le Roman du Genji se révèle comme une des sources littéraires majeures durant la période de formation de séquences longues, dans cette pratique poétique singulière qui pose comme principe fondamental de composition le changement continuel, donc la discontinuité 2 . Dans cette conception, dès le départ, très particulière quant au traitement du sens, on avait besoin d'un dispositif qui joue le rôle de fil conducteur, capable de justifier et motiver l'enchaînement des vers. Le procédé utilisé à l'origine pour cette fin est appelé fushimono 賦物 (thème inséré). Il consiste à employer en alternance deux séries thématiques de mots. Parmi ces séries, il en est une qui eut particulièrement du succès : celle des titres du Roman du Genji, qu'on faisait alterner avec la série des noms de provinces pour former des séquences de cent vers dites Genji kokumei renga 源氏国名連 歌. Ces thèmes sont généralement insérés de manière cachée, au moyen des homophones correspondants. Voici un exemple :

Est-ce pour faire sentir le froid que souffle le vent?

À cette cabane d'herbes que vous avez habitée

province du Musashi

de la triste prairie désertée ${ }^{3}$

chapitre Suma 
Samusa shire to ya Kaze wa fuku ran

さむさしれとや風は吹らむ

Suma-re-shi na Hitome kareno no Kusa no io

すま机しな人目か扎野入草の庵

Cette organisation thématique épuisa au bout d'un certain temps ses possibilités et fut délaissée au début du XIV siècle. Autrement dit, dès cette époque, on se mit à la recherche de nouveaux dispositifs permettant d'assurer l'enchaînement des vers. Fidèles à la tradition poétique focalisée singulièrement sur les mots et leurs relations codées, les poètes de renga explorèrent tous les documents reconnus comme classiques pour recenser des expressions pouvant enrichir et diversifier les réseaux de mots associés, ainsi qu'en témoigne Nijo Yoshimoto 二条良基 (1320-1388), l'une des plus hautes figures de la cour et grand amateur et théoricien du renga, qui réussit à le hisser au rang de littérature noble ${ }^{4}$.

3 De nouveau, le Roman du Genji s'imposa: on constitua des listes de mots du Genji, appelés Genji kotoba 源氏詞 ou Genji yoriai 源氏寄合 《associations codées tirées du Genji ", destinés à être utilisés pour l'enchaînement des vers. Le premier exemple fut le répertoire intitulé Hikaru Genji ichibu renga yoriai 光源氏一部連歌寄合 (Associations codées tirées de tout le Genji radieux) établi sur l'initiative de Yoshimoto en 1365. Voici un exemple de l'emploi que fait de ces mots une séquence tirée d'une série de mille vers appelée Mille vers sur la plaine violette (Murasakino senku 紫野千句) ${ }^{5}$ :

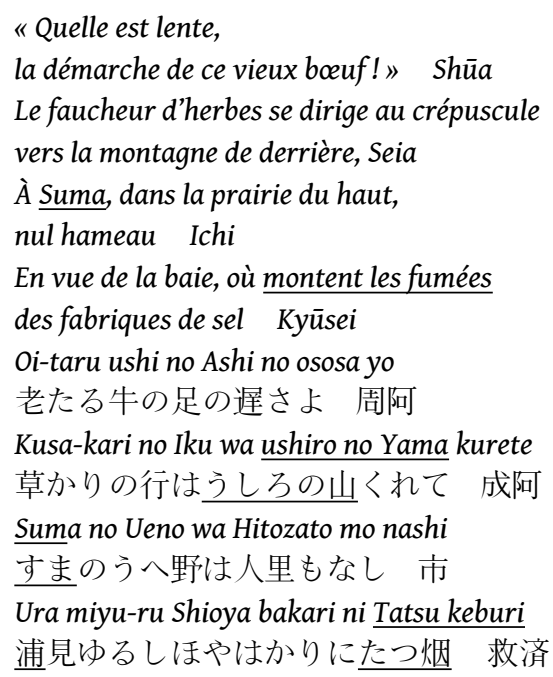

4 Les mots soulignés sont des Genji kotoba ${ }^{6}$. Ils proviennent d'un passage du chapitre Suma décrivant le Genji, frustré de se retrouver en exil dans la province de Suma, mais curieux tout de même des choses nouvelles qu'il aperçoit autour de lui :

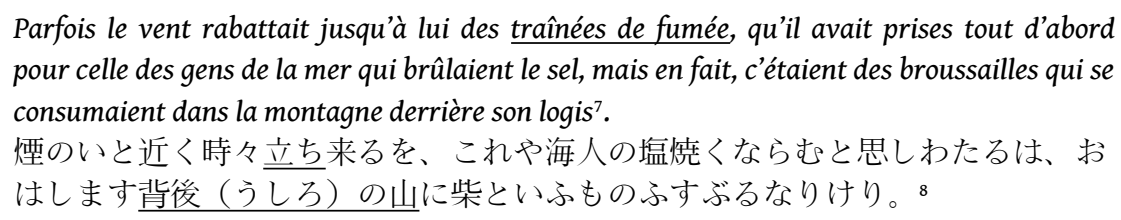

5 Les renvois au passage s'opèrent strictement sur le plan des mots, sans aucune allusion aux événements du roman. On part d'abord d'une scène champêtre où un paysan promène son bœuf chargé de foin. Se dresse derrière lui une montagne crépusculaire. Ce mot " ushiro no yama » rappelle aux participants un passage du Genji, et aiguillonne l'enchaînement vers l'emploi d'un autre mot appartenant à la liste : "Suma » sert ici comme un appel clair pour une composition recourant à un Genji kotoba - signal bien 
reçu par Kyūsei qui présente le sien en utilisant les mots «tatsu keburi ». Le toponyme permet en même temps un changement de paysage : on passe d'un arrière-pays désert à une vue marine dégagée d'où « montent les fumées ». La séquence n'a aucun rapport avec le dépaysement du prince Genji évoqué dans le roman ni avec la nostalgie qu'éprouve le personnage romanesque pour la vie de la capitale, nostalgie qu'il confiera une ligne plus loin dans un poème?

Voilà le premier type d'utilisation du Genji, entièrement focalisée sur les mots en tant que tels. Pour cette raison, on peut y voir une variante évoluée de l'emploi des titres des chapitres du Genji ${ }^{10}$. Mais, si les Genji kotoba sont apparus à cette période, c'est sans doute parce que les poètes ressentaient avant tout le besoin d'établir des références incontestables qui justifient l'enchaînement aux yeux de tous, et qui rendent possible le cheminement collectif.

7 La règle de base interdisant qu'un développement narratif déborde au-delà de deux vers, ce discours collectif est dominé par la contingence et se place à l'antipode d'une narration sous-tendue par la vision cohérente d'un ensemble. On passe d'un fragment à un autre, on se délecte de digressions continuelles. Mais la contingence pure étant un exercice impossible et sans doute dénué de sens, divers procédés motivant l'enchaînement ont été conçus. Ils sont essentiellement dominés par la pensée combinatoire et thématique : consigne d'espacement entre les séquences évoquant la même saison, restriction d'emploi pour certains mots... La codification des Genji kotoba fait partie de cette stratégie. Et comme nous venons de le voir, l'enchaînement à l'aide d'expressions tirées du Roman du Genji se justifie sans considération du fil narratif qui les réunit dans l'œuvre originale. Cette poétique, tournée vers les successions fragmentaires, a ainsi produit une curieuse situation où l'on se sert d'un vocabulaire tout en évacuant la dimension narrative qui est pourtant la raison d'être de ce vocabulaire. D'une manière imagée, on pourrait dire que les mots tirés du roman sont comme des fils d'or qu'on introduit de temps en temps pour donner de l'éclat à l'étoffe qu'on est en train de tisser. Le prestige littéraire qu'ils portent suffit à justifier leur emploi.

Dans beaucoup de cas, les Genji kotoba semblent être utilisés, comme dans cet exemple, uniquement pour motiver l'enchainement. Mais, même dans ce cas, ces mots ont le pouvoir de nous transporter dans l'univers du roman : le paysan qui promène un bœuf nous renvoie aux pêcheurs frustes que le prince rencontre pour la première fois à Suma; la vision d'une plage saisie par le regard d'un voyageur à celle du prince contemplant le paysage marin. Mais la relation de parenté reste ténue et empêche la formation d'un ensemble d'images cohérentes. Autrement dit, ce type d'emploi de mots " romanesques » a pour effet de créer un discours à deux niveaux : une ébauche de récit créée par les deux vers d'un enchaînement -- pour les deux derniers vers de la séquence citée plus haut, un voyageur découvre un paysage marin après avoir parcouru un arrière-pays -- et le souvenir de la situation romanesque à laquelle renvoient les mots insérés -- le Genji regardant les fumées qui montent. Les vagues images que tel mot du roman éveille dans l'esprit des participants ne s'intègrent pas directement au monde par définition fragmentaire des vers en chaîne. Elles flottent autour de lui comme des mirages. 


\section{Dimension narrative}

Les poètes de renga s'intéressaient aussi naturellement au potentiel narratif qu'offrait le Roman du Genji. Ainsi à un vers qui parle d'un attachement sentimental, Shōhaku 肖 柏 (1443-1527), un des plus talentueux poètes de renga, enchaîne un autre vers évoquant l'image de la jeune Murasaki tourmentée par les visions qui lui rappellent le Genji :

À toute heure, en tout lieu, je ne l'oublie pas :

M'adosser au pilier de cèdre, m'allonger sur le coussin la nuit, mouiller mes manches de larmes...

Tonikakuni tada Omoi wasure-zu

とにかくにた〉おもひ忘す

Maki-bashira Shitone ni yoru mo Sode nurete

槙はしらしとねによるも袖ぬれて11

Le vers est inspiré du passage de Suma ci-après :

La vue des endroits où il allait et venait, du pilier de cèdre auquel il avait coutume de s'adosser ne faisait que lui serrer le cœur ${ }^{12}$.

出で入りたまひし方、寄りみたまひし真木柱などを見たまふにも肮のみふたが

りて13

10 L'effet narratif est évident. Le contexte, la personnalité de l'héroïne, le lieu, ont beau ne pas être exprimés, ils sont là. Au lieu de les fixer en les détaillant, Shōhaku les libère dans un espace imaginaire où se croisent la poésie et le roman.

11 L'enchaînement suivant, réalisé par Shinkei心敬 (1406-1475), illustre également la fonction qu'un ouvrage narratif peut assumer dans la progression des vers :

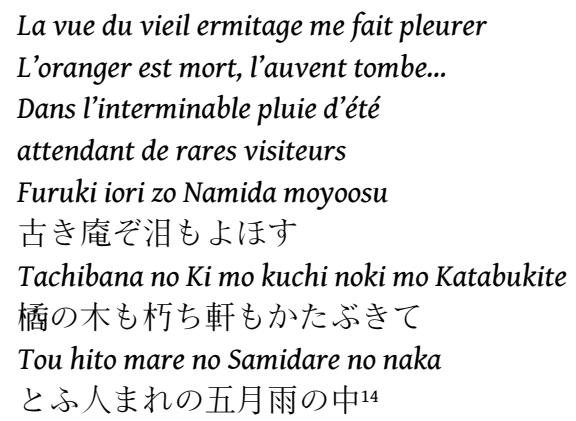

12 Comme le remarque Kaneko, le second vers précise l'aspect délabré de l'ermitage introduit par le premier vers. Selon lui, le troisième vers s'intègre à cette unité pour évoquer l'ensemble sous la pluie. Cependant, ce dernier vers, objecte-t-il, est trop dépendant du second, et, seul, ne présente pas une image claire du lieu ${ }^{15}$.

Il nous semble que Kaneko n'a pas bien saisi les marques romanesques qui relient les deux derniers vers : les mots tachibana et samidare. Rappelons qu'une dame surnommée «Hana chiru sato » (Séjour où fleurs au vent se dispersent), une maîtresse discrète du Genji, était étroitement associée aux fleurs d'oranger à cause du poème que le prince avait composé quand il lui rendit visite pendant une période de pluie, samidare.

Cédant à l'attrait du parfum de l'oranger

Voici le coucou qui recherche

le séjour où fleurs au vent se dispersent ${ }^{16}$

Tachibana no Ka o natsukashimi Hototogisu

Hana chiru sato o Tazunete zo tou ${ }^{17}$

槅の香をなつかしみほととぎす花散る里をたづねてぞとふ 
Il faudrait rappeler aussi que cette princesse, qui n'avait d'autre appui que le Genji, devait faire face à des difficultés matérielles après le départ de celui-ci à Suma. Sa demeure se dégrade sous la pluie interminable:

Sur le bord du toit délabré la davallie désormais prospère. À cette vue la rosée sur nos manches tombe dru

disaient-elles ${ }^{18}$, ce qui lui rappela qu'en effet elles ne devaient avoir d'autre protecteur que le grateron qui croissait à l'entour, et comme il avait appris d'autre part que, du fait des longues pluies, le mur de clôture s'était par endroits effondré... ${ }^{19}$

荒れまさる軒のしのぶをながめつつしげくも露のかかる袖かな

とあるを、げに葎よりほかの後見もなきさまにておはすらんと思しやりて、長 雨に築地所どころ崩れてなむと聞きたまへば20

Shinkei a donc transformé l'image d'un ermitage de montagne en recourant à celle de la résidence délabrée de Hana chiru sato qu'il avait empruntée au roman. Contrairement à la critique de Kaneko, l'intérêt de l'enchaînement réside précisément en ce glissement d'un lieu à un autre. Reprenons le dernier vers. Il explicite le moment (saison de pluie) et la situation (solitude), mais laisse vides les autres cases narratives. Les Genji kotoba suppléent implicitement les éléments narratifs manquants que sont, dans ce cas précis, le personnage et le lieu.

\section{Le Roman du Genji à travers le miroir du renga}

\section{Succès du chapitre Suma}

16 Quand les poètes de renga recherchaient des mots dans des ouvrages de référence en prose, ils ne s'intéressaient pas uniquement au Roman du Genji. Mais, comme on l'a vu, très vite cet ouvrage s'imposa. Dès le premier écrit sur les principes de composition, on le cite ${ }^{21}$. C'est le seul titre d'ouvrage en prose à être mentionné dans un recueil des règles du renga datant du début du XVI ${ }^{\mathrm{e}}$, qui reprend les pratiques en vigueur depuis la période de Yoshimoto tout en les réadaptant à la mode de l'époque. On y cite une remarque de Yoshimoto recommandant une dérogation en faveur de ce roman: les allusions aux poèmes célèbres et aux textes en prose ne doivent pas être prolongées, selon les règles du renga, au-delà de deux vers, mais, eu égard à la longueur du Roman $d u$ Genji, une séquence s'y référant doit se poursuivre sur trois vers ${ }^{22}$.

La mise en valeur du Roman du Genji se comprend aisément étant donné la qualité et la taille de l'ouvrage. Son importance est telle qu'on se réfère à lui sans même le nommer précisément, en se contentant de le désigner par le terme, "kano monogatari (ce [fameux] roman) ». Tout cela est naturel. Ce qui, en revanche, attire notre attention, est le succès du chapitre Suma. Dans la rubrique consacrée à la mise en garde contre des erreurs d'emploi, tous les Genji kotoba mentionnés proviennent de ce chapitre. Nous lisons: «Les lustrations à Suma tombent sur le premier jour du Serpent. Il faut les situer au printemps ", ou encore, «La saison de pluie à Suma est en été ${ }^{23}$ ». Ces courtes remarques suggèrent que l'emploi de ces mots était suffisamment fréquent pour mériter des précisions. Les séries de cent vers (hyakuin) ou de mille vers (senku千句) composées durant la période médiévale attestent la réalité de cette tendance: le recours à ce chapitre est beaucoup plus fréquent qu'aux autres. Pourquoi spécifiquement le chapitre Suma? 
Signalons tout d'abord que ce chapitre était déjà très apprécié des poètes de waka, à commencer par Fujiwara no Teika藤原定家 (1162-1241), considéré durant toute la période médiévale comme le maître incontesté en matière de poésie ${ }^{24}$.

Ce chapitre possède plusieurs éléments qui peuvent séduire les sensibilités forgées par la tradition poétique. Tout d'abord, Suma fait partie des toponymes poétiques, utamakura歌枕, vocabulaire privilégié pour la composition du waka. Le terme en lui-même est particulièrement évocateur ${ }^{25}$. D'autre part, du point de vue des grands thèmes poétiques, le chapitre est riche en sujets d'inspiration : saisons, voyage et amour. En fait de saisons, thématique centrale du waka, mis à part l'été, un peu en retrait, le chapitre, fidèle à l'esthétique de la poésie, déploie une succession de scènes mettant en valeur les fleurs de cerisier pour le printemps et la lune pour l'automne. L'exil du Genji l'associe évidemment au voyage ${ }^{26}$ et au sentiment de précarité qui l'accompagne. L'amour malheureux, sentiment-type du waka, est vécu par tous les personnages principaux du chapitre Suma à cause de la séparation forcée qui résulte de l'exil du héros.

Le renga, fondé sur une esthétique de la diversité, affectionne le déplacement, le voyage. L'emploi de toponymes est un moyen pratique pour introduire un changement de lieu, comme nous l'avons vu. L'éventail varié des saisons facilite le changement de temps. Pour toutes ces raisons, le chapitre Suma constitue une réserve précieuse où puiser pour la conception des vers.

21 Mais le succès du chapitre Suma est aussi lié à une autre caractéristique du chapitre. Comme le signale Takada Hirohiko dans son article, Suma constitue une frontière entre la zone sous l'emprise directe du pouvoir impérial et les provinces de l'Ouest qui peuvent s'en émanciper. Mais dans ce chapitre la frontière ne semble pas être uniquement politico-géographique. Car la présence même du Genji dans cette zone décentrée crée une frontière à la fois vis-à-vis des hommes et de la nature. Ce personnage, incarnant la quintessence du raffinement, est pour la première fois en contact direct avec un univers qui échappe à la culture de la cour. Le texte insiste à plusieurs reprises sur la disparition de la distance entre ces deux mondes :

Les pitoyables conditions dans lesquelles vivait le Prince permettaient à un homme de cette sorte (un messager apportant d'Ise une lettre) de l'approcher ${ }^{27}$

かくあはれなる御住まひなれば、かやうの人もおのづからもの遠からで...28

Seul éveillé, quand il souleva la tête pour entendre l'ouragan qui faisait rage aux quatre horizons, il eut le sentiment que les vagues déferlaient à son chevet... ${ }^{29}$

独り目をさまして、枕をそばだてて四方の嵐を聞きたまふに、波ただここもと に立ちくる心地して ${ }^{30}$

Les rayons blancs de la lune pénétraient dans la chambre de ce séjour précaire, qu'ils éclairaient jusqu'au fond. De sa couche, il apercevait [à travers des failles du toit] le ciel de la nuit profonde.

月いと明うさし入りて、はかなき旅の御座所は奥まで隈なし。床の上に、夜染 き空も見ゆ。31

Les ustensiles rassemblés pour son usage étaient des plus sommaires, et l'on voyait dans tous ses détails la pièce où il séjournait. [...] Des gens de la mer apportaient des coquillages qu'ils venaient de pêcher; il les fit appeler pour les voir de près. Il les interrogea sur la manière dont ils passaient l'année sur ces rivages, et ils lui dirent leurs travaux et leurs peines. Dans leur informe baragouin, c'étaient les mêmes mouvements du cœur qu'ils exprimaient, se ditil, ému ${ }^{32}$.

取り使ひたまへる調度どもかりそめにしなして、御座所もあらはに見入れら

る。[...] 海人ども漁りして、貝つ物持て参れるを召し出でてご覧ず。浦に年経 るさまなど問はせたまふに、さまざま安げなき身の愁へを申す。そこはかとな くさへづるも、心の行く方は同じこと、何かことなるとあはれに見たまふ。33 
Le prince est donc surexposé aux regards des hommes et aux puissances de la nature. Sa chambre n'est plus dans une résidence somptueuse entourée de murs. Son intimité est visible de l'extérieur. Le monde raffiné qu'il représente se rétrécit aux dimensions de sa propre personne, ce qui le met en relation directe avec une population qu'il n'aurait jamais eu l'occasion de rencontrer en d'autres circonstances. Les deux visages de la nature -- l'un, riant et raffiné, représenté par le jardin aménagé par le Genji, l'autre, sauvage, représenté par les vagues déferlantes -- se juxtaposent également sans transition. Dans ce sens, le chapitre est l'illustration même de la coprésence du ga 雅 (style noble) et du zoku俗 (style bas ${ }^{34}$ ), caractéristique de la nouvelle esthétique qui distingue le renga du waka. Pour la même raison, les chapitres Yūgao (La belle-de-jour) et Yomogiu (L'impénétrable armoise) ont également eu du succès auprès de poètes de renga. Cependant, ces épisodes ne constituent pour le prince que de petites escapades. Par contre, la vie à Suma forge son destin et détermine le cours de l'histoire.

Ajoutons que le succès de Suma provient sans doute également de son contenu narratif très simple. Une fois arrivé à Suma, le Genji n'a plus d'activités significatives. Il ne fait qu'endurer la solitude dans un milieu rustique. Cette simplicité narrative aurait facilité le recours à ce chapitre dans des réunions où se côtoient des participants de niveaux de culture inégaux.

\section{La dimension mythologique}

L'épisode de Suma se résume à la réclusion du Genji en ce lieu. Mais, contrairement à cette simplicité apparente, sa lecture nous plonge dans un espace complexe. Quand le héros s'installe dans l'immobilité, le monde se met à s'activer autour de lui comme si cette inaction avait un pouvoir catalytique. C'est en fait le Genji lui-même qui déclenche le mouvement. Avant son départ de la capitale, il multiplie les déplacements, les scènes d'adieux se répètent et se succèdent, formant un effet de miroir. À Suma, le va-et-vient des messagers prend le relais, la romancière introduit des scènes situées à la capitale, et ces deux lieux opposés tendent à s'interpénétrer. Plus précisément, à cause de la présence du prince, l'endroit décentré se transforme peu à peu en un point central vers lequel les mouvements convergent : ceux du messager qui vient d'Ise, de l'adjoint principal du gouvernement général du Kyūshū en route pour la capitale, et de l'ami intime venu de la capitale lui rendre visite ${ }^{35}$.

Cependant, l'acteur principal est la nature qui se déchaîne à la fin du chapitre. L'orage s'abat sur Suma, détruit la résidence du prince, et atteint son paroxysme. Elle fait rage au sein même de la capitale, abolissant ainsi la distance qui sépare les deux lieux.

Depuis les commentaires anciens, on signale les éléments surnaturels et mythologiques du chapitre Suma. Outre les références mythologiques invoquées, ce caractère réside à nos yeux dans cette mise en valeur de la nature comme levier narratif. Sous l'effet de l'orage, la cloison entre le monde humain et un autre monde tombe : une apparition commence à hanter le rêve du prince, le contour des choses devient incertain :

Or voici que, venu à grand peine de la Deuxième Avenue [la résidence de Murasaki], se présenta un messager en piteux état, trempé jusqu'à l'os. Ce rustre que, s'il l'avait croisé sur son chemin, il eût hésité à tenir pour un être humain, et que l'on eût sur l'heure chassé de sa présence, lui inspira une sympathie qui lui fit reconnaître, avec horreur, à quel degré de déchéance il était tombés ${ }^{36}$. 
二条院よりぞ、あながちに、あやしき姿にてそぼち参れる。道交ひてだに、人

か何ぞとご覧じわくべきもあらず、まず追ひ出ひつべき賤の男の睈ましうあは

机に思さるるも、我ながらかたじけなく屈しにける心のほど思ひ知らる。37 événements, et place le Genji d'emblée dans sa relation sociale : il reçoit la veille de son 
départ son frère et son meilleur ami, vêtu de la casaque (nōshi) sans motif (mumon無紋), signe de sa disgrâce infligée par la cour. En d'autres termes, l'image du Genji est organisée ici, d'abord à travers les regards virtuels ${ }^{39}$ de ces visiteurs, et se voit conférer une dimension dépassant la vie proprement personnelle du prince. Ce n'est pas tout. À travers la citation intégrale de la scène du miroir, l'Ekotoba saisit les personnages à travers un autre type de regard: celui réflexif, qui se referme sur lui-même. C'est d'abord le regard que le Genji porte sur son propre reflet dans le miroir et ensuite celui de la princesse en larmes qui le contemple. Grâce au traitement attentif des éléments narratifs, l'Ekotoba réussit à dégager l'opposition entre ces deux types de regards et souligne la solitude des deux personnages.

Les citations des poètes de renga, le Kokagami et le Shijin, amplifient la seconde partie, la scène du miroir. Le Kokagami se concentre sur la seule personne du Genji, ne gardant pour la jeune Murasaki que l'expression, « hashira gakure (à l'ombre d'un pilier) ». Le Shijin se positionne à un degré supérieur de condensation. La citation, qui fait abstraction des actes précédant l'échange des poèmes, se rapproche d'un kotoba-gaki詞 書, bref texte ayant pour rôle de présenter les circonstances de composition d'un poème. Mais, en même temps, comparé au Kokagami, le texte du Shijin, en se focalisant sur l'échange de regards et de poèmes, équilibre le poids respectif de chacun des deux acteurs de la scène.

Dans tous les cas, l'échange de poèmes n'est jamais négligé, et ces trois citations décrivent une configuration concentrique, qui témoigne de la place privilégiée des poèmes dans la réception et la transmission du Roman du Genji. Il faudrait à ce propos insister sur le caractère éminemment subjectif du waka, qui tend à mettre en scène un instant d'une existence avec toute sa densité et à détacher ce moment du déroulement spatio-temporel des faits. De l'Ekotoba au Shijin, en passant par le Kokagami, s'opère progressivement le détachement des gestes des contextes narratifs. Le Kokagami supprime la motivation de l'acte du Genji -- se regarder dans le miroir pour accueillir les visiteurs -- précisée dans l'Ekotoba. Le Shijin va encore plus loin en supprimant le geste même de se regarder dans le miroir pour arranger sa coiffure - que conservait la citation du Kokagami, pour ne garder que le reflet dans le miroir, qui se présente ainsi comme un effet sans but et sans origine. 


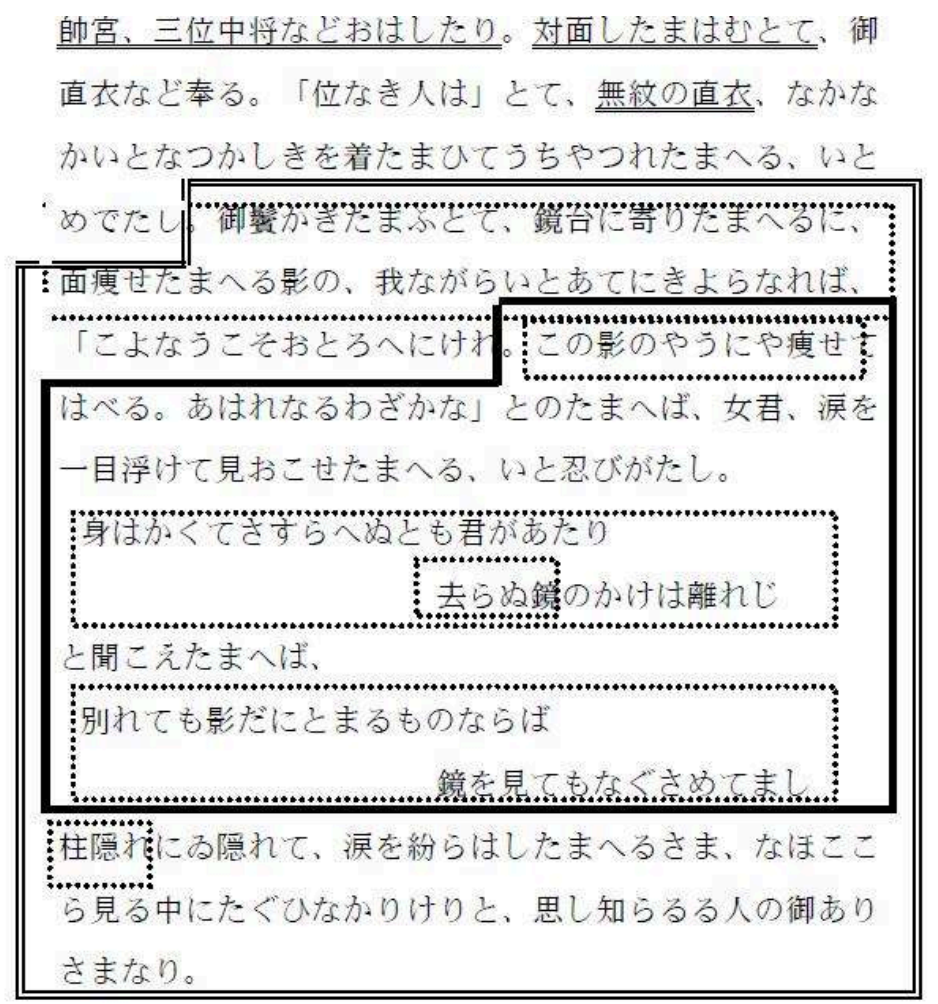

御子は、あはれなる御物語聞こえたまひて、暮るるほどに帰りたまひ ぬ。 40

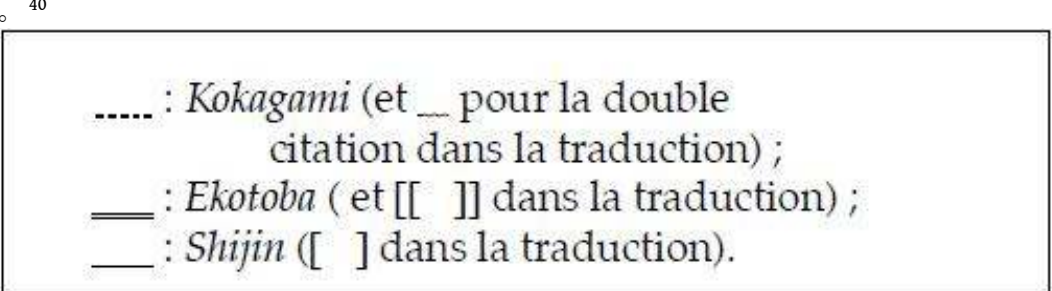

Le Prince Gouverneur Général et le Commandant du Troisième Rang s'étaient fait annoncer. Pour les recevoir, il revêtit une casaque. Et parce qu'il n'avait plus de rang, il mit une casaque sans dessin, fort seyante, tenue modeste qui ne le rendit que plus aimable. [[Pour démêler les cheveux de ses tempes, il s'assit devant le miroir, et l'image de son visage émacié était, il dut en convenir lui-même, d'une rare distinction.

- Me voici tombé bien bas, [amaigri comme cette image. Je dois faire pitié... dit-il. La dame, les larmes aux yeux, le considérait, ce qu'il ne put supporter.

Je m'en irai errant au loin, mais mon image

Fixée dans ce miroir ne vous quittera pas dit-il. Elle:

Après votre départ, si seulement cette image restait

Je me consolerais en regardant le miroir]

$E t$, à l'ombre d'un pilier, elle dissimulait ses larmes; il la contemplait, se disant que chacun de ses gestes la distinguait entre toutes.]] Le Prince Gouverneur Général se répandit en propos touchants avec lui et le quitta à la tombée du jour ${ }^{41}$.

Bien qu'encore un peu schématique, méritant d'être approfondie, l'analyse que nous avons menée nous permet de constater ceci : la superposition de ces trois extraits fait ressortir deux écritures qui sous-tendent le texte du roman, le récit à poèmes (歌物語) qui est la forme développée du kotoba-gaki et le récit avec illustrations. Ces deux formes d'écriture ont en commun leur prédilection pour les scènes, mode de représentation fondée sur un temps qui se dilate et au centre duquel rayonnent les poèmes, les waka qui sont l'expression de la subjectivité par excellence ${ }^{42}$. 
L'originalité du Roman du Genji consiste à avoir refondé la prose comme espace d'épanouissement de la subjectivité pure ${ }^{43}$ au même titre que le waka, au lieu de simplement l'encadrer comme le font un grand nombre de récits à poèmes. Mais, sans doute, comme le signale à juste titre Takada, la vraie originalité de cet ouvrage réside dans le fait que cette écriture s'appuie sur un dispositif narratif très solide. De même que le travail du peintre requiert des éléments concrets, l'Ekotoba s'appuie sur la situation narrative. Grâce à sa citation, on sait pourquoi le Genji se regarde dans le miroir : il se prépare à accueillir les visiteurs et entre autres, son frère, prince impérial. Autrement dit, pendant que le Genji refait sa coiffure et échange des poèmes avec Murasaki, son frère l'attend gentiment, ce dont le Kokagami et le Shijin font abstraction. Ces derniers se concentrent sur l'effet envoûtant de l'image et accentuent encore l'hypertrophie propre à la scène.

La marche du temps est inégale dans toute narration. La description la ralentit tandis que l'ellipse dans l'agencement des événements l'accélère. Ce qui frappe dans ce passage du roman est la juxtaposition des deux écritures opposées qui s'enchainent sans aucune mise en perspective : la scène, fondée sur le temps qui se dilate, est relayée sans transition par une écriture neutre, qui contracte le temps : « Le Prince Gouverneur Général se répandit... ». Le décalage entre ces deux temps, dilatation et contraction, renforce la puissance de l'image. Comme on l'a vu, ces deux éléments, la scène du miroir et la visite du prince impérial, sont étroitement liés sur le plan du récit : si l'on parle du miroir, c'est parce qu'il y a une visite. L'écriture s'appuie ainsi sur une trame narrative très solide, tout en faisant rayonner des scènes plus ou moins autonomes. Le texte maintient globalement l'équilibre entre les deux niveaux de représentation, en les juxtaposant, comme dans cet exemple, ou en les fondant ailleurs en une seule écriture, comme c'est le cas dans le passage consacré à la visite du Genji chez Hana chiru sato avant son départ à Suma. La scène de cette belle nuit éclairée par la lune, tout en nous dévoilant la condition précaire de la princesse, annonce déjà la vie du Genji en exil.

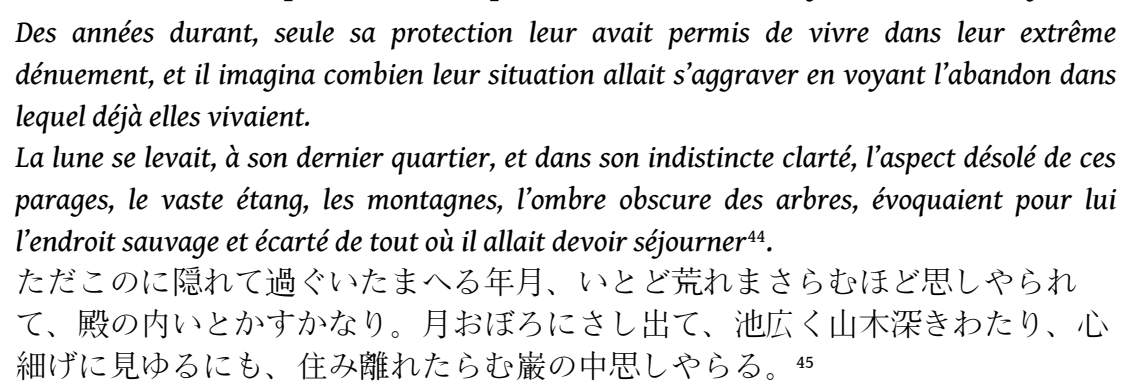

Les Genji kotoba et les citations de passages tendent à amplifier l'hypertrophie des scènes. Mais, en même temps, leur excès éclaire indirectement la dualité de l'écriture du Genji, qui est peut-être de l'invention de cette romancière hors du commun.

\section{Une écriture de la dissémination}

39 Ce qui nous paraît remarquable dans cette écriture, c'est que l'un des éléments de la double structure que nous venons de dégager, à savoir: la scène plus ou moins autonome, se comporte d'une manière paradoxale. Tout en perturbant le déroulement narratif, il sert d'une manière originale à le renforcer. Les Genji kotoba concourent à souligner cette structure complexe. Prenons, par exemple, yomo no arashi四面の嵐 (l'ouragan qui fait rage aux quatre horizons). Le terme apparaît dans un des passages 
les plus célèbres du chapitre, qui décrit la solitude et la détresse du Genji ${ }^{46}$. Cette expression pointée, cataloguée comme Genji kotoba, attire notre attention quand elle apparaît à un autre endroit. Or le terme fait partie justement d'un poème du chapitre Sakaki (L'arbre sacré), chapitre qui précède directement Suma. la capitale :

41 Dans Suma, le prince envisage de reprendre sur le lieu de son exil cette existence vouée au bouddhisme qu'il avait goûtée pendant son séjour au monastère et qu'il avait quittée à regret. C'est en partie pour cette raison qu'il renonce à l'idée d'emmener Murasaki dans son exil. Ainsi, ils vont vivre séparés durant cette période. Le passage de L'arbre sacré où apparaît yomo no arashi préfigure donc la situation du chapitre Suma. L'orage qui se déchaîne à la fin de ce chapitre commence à gronder déjà dans le chapitre précédent.

42 Le mécanisme de renvois entre les expressions, tout en conférant une épaisseur lyrique au récit, sert donc à structurer le récit lui-même, selon un système qu'on pourrait qualifier de « dissémination ». En effet, la lecture du Roman du Genji nous donne parfois l'impression que les expressions clés y sont distribuées de façon à se répondre au-delà de tout contexte narratif. En même temps, comme le montre l'exemple de l'« ouragan qui fait rage aux quatre horizons ", ces éléments saillants nouent les épisodes avec un fil invisible, en deçà de l'agencement narratif. Les Genji kotoba nous aident à cerner cette écriture d'une souplesse et d'une efficacité redoutables. Ils nous apprennent que le texte du Roman du Genji constitue en quelque sorte un faisceau de chemins qui partent dans de multiples directions, et en même temps un point de concentration vers lequel convergent divers chemins.

\section{NOTES}

1. Voir Sumie Terada, «La prose dans le contexte de la poésie », in Éloge des sources, Arles, éd. Philippe Picquier, 2004, pp. 141-166.

2. La composition est réalisée collectivement : les participants se rassemblent et proposent, tour à tour, des vers qu'ils conçoivent sur place pour former généralement un hyakuin 百韻, une séquence de cent vers. Chaque vers dans cet enchaînement effectue une double opération, continue et discontinue: il forme une unité cohérente avec le vers qui le précède tout en dissociant ce dernier du vers auquel il succède.

3. Itai senku, Fu Genji kokumei renga daiichi 異体千句 賦源氏国名連歌第一 (Mille vers de formes particulières, première centaine [hyakuin], thèmes : le Roman du Genji et les noms de provinces), $64^{\mathrm{e}}$ et $65^{\mathrm{e}}$ vers, Senku renga shū 3, coll. "Koten bunko », éd. Shimazu Tadao et al., 1981, p. 228. Cette série de mille vers fut composée en 1456 par Kanisawa Gen.i 金（蟹）沢下野守入道源意， guerrier entré en religion, vassal de Yamana et gouverneur de la province de Shimotsuke, sous l'influence de la faveur nouvelle dont jouissent les anciennes pratiques à l'époque. 
4. Pour le témoignage de Yoshimoto, voir S. Terada, op. cit., p. 167.

5. Cette séance de renga fut organisée au plus tard en 1370 avec la participation de Yoshimoto et de Kyūsei (Gusai) 救済 (1282 ?-1376 ?), un des plus grands poètes de renga du XIV siècle. Les vers cités sont les $76^{\mathrm{e}}-79^{\mathrm{e}}$ de la cinquième centaine, coll. «Koten bunko ", Senku renga shū 1, éd. Shimazu Tadao et al., 1978, p. 108.

6. Diverses listes furent constituées au fil du temps et le nombre ainsi que le choix des mots varient considérablement des unes aux autres. Pour ce passage, nous nous sommes référée à la liste proposée par le Hikaru Genji ichi-bu renga yoriai. Les pointillés signalent d'une part les expressions qui ne figurent pas dans la liste et d'autre part des termes qui figurent bien dans la liste, mais se rapportent à d'autres passages du Genji.

7. Le Dit du Genji, tome 1, trad. René Sieffert, p. 272.

8. Genji monogatari, Shinpen Nihon koten bungaku zenshū (ci-après SNKBZ), Shōgakukan, tome 2, 1995, pp. 207-208.

9. Ce poème est analysé ici même dans l'article de Michel Vieillard-Baron. http:// cipango.revues.org/596

10. Ichijō Kanera (1402-1481), le petit-fils de Yoshimoto, qui joua un rôle analogue à ce dernier, déplore la tendance consistant à utiliser à tort et à travers les Genji kotoba dès qu'il s'agit d'enchaîner un vers à un autre contenant un titre de ce roman (cité par Ii Haruki, « Renju gappeki shū ni mi-rareru Genji yoriai - Genji kokagami, Hikaru Genji ichibu renga yoriai, Genji monogatari nai renga tsukeai nado to no kanren »-, in Renga to sono shūhen, Hiroshima chūsei bungaku kenkyūkai, Hiroshima, 1967, p. 426).

11. Shunmusō chū, in Renga kochūshaku shū, Kaneko Kinjirō (éd.), Kadokawa shoten, 1979, p. 289.

12. Sieffert, op. cit., p. 262.

13. SNKBZ, op. cit., p. 190.

14. Ōnin gannen natsu Shinkei dokugin yamanani hyakuin 応仁元年夏心敬独吟山何百韻 (Cent vers en solo de Shinkei ayant comme thèmes insérés des mots comprenant «montagne ", composés durant l'été de la première année de l'ère Ōnin [1467]), vers $88^{\mathrm{e}}-90^{\mathrm{e}}$. Shinkei représente avec Sōgi 宗祗 (1421-1501), le plus célèbre poète de renga et maître de Shōhaku, la poésie de l'époque médiévale. Précisons que le thème inséré (fushimono) était devenu à cette époque un dispositif accessoire qui ne s'appliquait plus qu'aux premiers vers.

15. Shinkei no seikatsu to sakuhin, Kaneko Kinjirō, Ōfūsha, 1982, p.315-316. Kaneko associe 《tachibana » avec un épisode du Tsurezure gusa 徒然草 (Les heures oisives), chapitre 11.

16. Sieffert, op. cit., p. 245.

17. SNKBZ, op. cit., p. 156 (chapitre Hana chiru sato).

18. Elle vit avec sa sœur, une des épouses du défunt empereur, père du Genji.

19. Sieffert, op. cit., p. 265.

20. SNKBZ, op. cit., p. 196.

21. Une remarque dans le Yakumo misho 八雲御抄 (Traité des nuées divines) sur l'emploi d'un titre de chapitre du roman comme thème inséré se rencontre à la rubrique consacrée au renga. Cet ouvrage monumental consacré à la poésie japonaise fut rédigé par l'empereur Juntoku 順徳 (1197-1242) dans les années 1220, puis complété par la suite (Nihon kagaku taikei, tome sup. 3, Kazama shobō, 1972, p. 205).

22. Renga shinshiki tsuika narabi ni shinshiki kon. an tō 連歌新式追加站新式今案等 (Nouvelles règles du renga complétées et réadaptées), compilé en 1501 par Shōhaku. Shōhaku lui-même n'était pas convaincu du bien-fondé de ce traitement d'exception (Shinkō gunsho ruijū, tome 13, vol. 306, p. 714).

23. Apparemment, il existait à propos de ces matières des opinions divergentes. Nous lisons tout de suite après un ajout qui dément cette affirmation (Ibid., p. 719).

24. Sur l'intérêt de Teika pour ce chapitre, voir S. Terada, op. cit., p. 156. 
25. Sur la richesse des réseaux poétiques gravitant autour de ce toponyme, voir ici-même l'article de Takada Hirohiko.

26. Précisons que le mot たび avait la signification d'un séjour en dehors de la maison où l'on vit habituellement.

27. Sieffert, op. cit., p. 264.

28. SNKBZ, op. cit., p. 195.

29. Sieffert, op. cit., p. 195.

30. SNKBZ, op. cit., p. 199.

31. Ibid., p. 266.

32. Sieffert, op.cit., p. 275. Le sujet des deux dernières phrases dans la traduction est le Commandant du troisième rang, ami intime du Genji qui lui rend visite au cours de son exil. Le commentaire de l'édition Shinchō (tome 2, p. 251) considère que le sujet est plutôt le Genji et son ami, ce qui nous paraît aussi plus plausible.

33. SNKBZ, op. cit., p. 213-214.

34. Dans la poésie japonaise, le ga (noble ou raffiné) représente l'esthétique orthodoxe du waka, et le zoku (bas, familier, trivial ou vulgaire) renvoie à tout ce qui est exclu de cette poésie noble. Le renga s'est développé au départ comme un genre léger destiné au divertissement, récupérant ce qui est rejeté par le waka, à commencer par le vocabulaire de la vie quotidienne (zokugo) et les composés sino-japonais, appelés haigon 俳言.

35. Notons au passage que ce sont des lieux hautement symboliques. On pourrait ajouter à cette liste Matsushima, qui représente l'Est du Japon. Ce toponyme apparaît dans les poèmes échangés entre le Genji et Fujitsubo, épouse impériale de feu son père, avec laquelle le Genji a eu une liaison secrète.

36. Sieffert, op. cit., p. 279.

37. Chapitre Suma, SNKBZ, op. cit., p. 224.

38. Rappelons que Sōgi était le premier spécialiste du Roman du Genji de son époque.

39. Nous disons "virtuels", dans la mesure où ils ne sont pas mentionnés explicitement, mais simplement suggérés dans le texte.

40. SNKBZ, op. cit., p. 173.

41. Sieffert, op. cit., p. 252-253. La traduction est partiellement remaniée.

42. Sur ce point, voir Jacqueline Pigeot, «Autour du waka », in Questions de poétique japonaise, Puf, 1997, p. 1-8, et S. Terada, «La prose dans les anthologies de poèmes », in Anthologie poétique en Chine et au Japon, Extrême-Orient Extrême-Occident, $\mathrm{n}^{\circ}$ 25, 2003, p. 99-119.

43. Voir par exemple le passage cité par Takada http://cipango.revues.org/589.

44. Sieffert, op. cit., p. 253. La mise en page adoptée dans la traduction souligne la structure du texte original. En effet, contrairement aux principales éditions japonaises actuelles, Sieffert sépare le passage en deux parties distinctes par l'introduction du changement de ligne : partie purement narrative et partie dominée par la description du paysage.

45. SNKBZ, op. cit., p. 174.

46. Voir l'article de Takada pour l'analyse de ce passage http://cipango.revues.org/589.

47. Sieffert, op. cit., p. 226 (traduction remaniée).

48. SNKBZ, op.cit., p. 118. 


\section{RÉSUMÉS}

Devenu une référence, le Genji contribue au développement du waka et du renga dans le Japon médiéval. Le chapitre «Suma » est particulièrement caractéristique de la nouvelle esthétique qui distingue alors ces deux formes poétiques.

Genji monogatari soon became a standard book and contributed to the development of waka and renga in Middle Age Japan. The chapter entitled "Suma" is particularly characteristic of the new aesthetic that distinguished these two poetic forms.

\section{INDEX}

Thèmes : littérature

キーワード : Genji monogatari 源氏物語, monogatari 物語, renga 連歌, waka 和歌, Murasaki Shikibu 紫式部 (v. 973-v. 1014 ou 1025), shi 詩, Suma 須磨, Heian jidai 平安時代 （794-1185), bungaku 文学

Index géographique : Suma

Keywords : Genji monogatari, Heian, Literature, monogatari, renga, waka, Tale of Genji, Murasaki Shikibu (v. 973-v. 1014 or 1025), Poetry, Suma

Mots-clés : Genji monogatari, monogatari, renga, waka, Dit du Genji, Murasaki Shikibu (v. 973-v. 1014 ou 1025), poésie en chaîne

Index chronologique : Heian 